\title{
Use of Alpha ${ }_{2}$ Adrenoreceptor Agonists and Antagonists in the Functional Assessment of the Sympathetic Nervous System
}

\author{
David Robertson, Michael R. Goldberg, Che-Se Tung, Alan S. Hollister, and Rose Marie Robertson \\ Autonomic Dysfunction Clinic, Departments of Medicine and Pharmacology, Vanderbilt Medical School, Nashville, Tennessee 37232
}

\begin{abstract}
We studied the effects of clonidine, an $\alpha_{2}$-adrenoreceptor agonist, and yohimbine, an $\alpha_{\mathbf{2}}$-adrenoreceptor antagonist, on blood pressure, heart rate, and plasma catecholamines in 12 patients with autonomic dysfunction. Clonidine (0.1 $\mathrm{mg}$, orally) lowered blood pressure $18 \pm 3$ torr in six subjects and raised it $5 \pm 1$ torr in six. The change in blood pressure in response to this dose of clonidine was inversely proportional to the supine level of norepinephrine $(P<0.05)$. Yohimbine (4-64 $\mu \mathrm{g} / \mathrm{kg})$ raised plasma norepinephrine and blood pressure in six patients with degenerative autonomic dysfunction. Yohimbine also attenuated by $50 \%(P<0.05)$ the hypotensive response to head-up tilt of patients with degenerative autonomic dysfunction.

Clonidine depends upon postjunctional hypersensitivity and the degree of autonomic insufficiency to elicit its pressor response. In contrast, the pressor response to yohimbine is related to the capacity of the sympathetic nervous system to be activated and release norepinephrine. If plasma norepinephrine levels following yohimbine administration are monitored, the biochemical and hemodynamic response to the drug may provide a sensitive indication of the capacity of the sympathetic nervous system to be activated in patients with autonomic dysfunction.
\end{abstract}

\section{Introduction}

The autonomic nervous system regulates the body's hemodynamic response to postural and other stresses. In autonomic failure, the body is unable to respond to these stresses and this results in marked functional impairment, primarily manifested as orthostatic hypotension. Such autonomic abnormalities are not rare; they occur frequently in diabetes mellitus and amyloidosis and accompany the use of many antihypertensive and psychiatric drugs. Less commonly, autonomic dysfunction is primary, affecting older individuals and severely limiting their activities. Such patients may have autonomic dysfunction associated with multiple nonautonomic neurologic defects (Shy-

Portions of this work were presented at the Annual Meeting of the American Federation for Clinical Research, Washington, DC, 30 April-2 May 1983.

Dr. Tung's present address is Pharmacology Department, National Defense Medical Center, Taipei, Taiwan, Republic of China. Address correspondence to Dr. David Robertson, Autonomic Dysfunction Clinic, Division of Clinical Pharmacology, Vanderbilt Medical School, Nashville, TN 37232.

Received for publication 24 December 1985 and in revised form 17 April 1986

J. Clin. Invest.

(C) The American Society for Clinical Investigation, Inc.

0021-9738/86/08/0576/06 \$1.00

Volume 78, August 1986, 576-581
Drager syndrome or multiple system atrophy [MSA] ${ }^{1}$ or they may lack nonautonomic signs and symptoms (Bradbury-Eggleston syndrome or idiopathic orthostatic hypotension [IOH]).

Treatment of these patients remains empiric, relying on maneuvers or agents that increase effective intravascular volume and/or on pressor agents (1-3). Although many careful studies have been performed in these subjects, many questions about their altered cardiovascular and autonomic physiology remain unanswered. In the evaluation of these patients, documentation of the absence of secondary causes of autonomic dysfunction and confirmation of the magnitude of the deficiency in sympathetic outflow through catecholamine determinations, reflex testing, and pharmacological sensitivity testing $(1,4-6)$ may allow more specific therapy.

In recent years, $\alpha_{2}$-adrenoreceptors in the central nervous system have been found to be important in the regulation of sympathetic outflow in normal man and are therapeutic targets for centrally active antihypertensive agents $(7,8)$. The function of $\alpha_{2}$-receptors in patients with autonomic dysfunction has not been extensively studied (9). It is known that some severely affected patients with $\mathrm{IOH}$ can manifest a pressor response to the $\alpha_{2}$ partial agonist, clonidine, presumably because they have little spontaneous sympathetic outflow and because they are supersensitive to pressor agents (9). It is not known whether, or to what extent, $\alpha_{2}$-receptors contribute to the disability of less severely affected patients. It is possible that residual sympathetic activity in these less severely affected patients could be inhibited by clonidine (9), leading to a depressor effect. In this setting, the facilitation of the residual sympathetic activity by $\alpha_{2}$ blocking agents often results in functional improvement $(7,10)$. We have therefore undertaken an investigation of the effects of the $\alpha_{2}$ partial agonist, clonidine, and the $\alpha_{2}$ antagonist, yohimbine, in 11 patients with autonomic dysfunction. Three patients had associated mild extrapyramidal defects (MSA) while eight lacked nonautonomic involvement (IOH). A 12th patient with a selective defect in noradrenergic function was also studied. This patient was unable to synthesize norepinephrine from dopamine in the peripheral sympathetic noradrenergic terminals (11).

\section{Methods}

All patients were recruited from the Vanderbilt Autonomic Dysfunction Clinic and studied at the Elliot V. Newman Clinical Research Center. Experimental procedures and consent forms were approved by the Vanderbilt Committee for the Protection of Human Subjects. Results of the diagnostic evaluation of these subjects are summarized in Table I. All subjects had diminished or absent pressor reflexes, subnormal or lownormal levels of plasma norepinephrine, relatively reduced response to tyramine infusions, and increased sensitivity to phenylephrine and isoproterenol $(6,7,12,13)$. The patient with the selective noradrenergic

1. Abbreviations used in this paper: $\mathrm{IOH}$, idiopathic orthostatic hypotension; MSA, multiple system atrophy; MTT, maximum tolerated tilt. 
Table I. Clinical Findings in Patients with Autonomic Dysfunction

\begin{tabular}{|c|c|c|c|c|c|c|c|}
\hline Patient & Age/sex & Diagnosis* & Supine plasma NEł & Cold pressor§ & Tyr-25" & Phe-25" & Iso-25" \\
\hline 1 & $62 / \mathrm{F}$ & $\mathrm{IOH}$ & 34 & 0 & 4,400 & 30 & 0.4 \\
\hline 2 & $68 / \mathrm{M}$ & $\mathrm{IOH}$ & 42 & 0 & 4,000 & 25 & 0.4 \\
\hline 3 & $69 / \mathrm{F}$ & $\mathrm{IOH}$ & 34 & 0 & 5,200 & 40 & 0.3 \\
\hline 4 & $74 / \mathrm{M}$ & $\mathrm{IOH}$ & 27 & 0 & 6,000 & 40 & 0.4 \\
\hline 5 & $62 / \mathrm{M}$ & $\mathrm{IOH}$ & 68 & 1 & 2,300 & 25 & 0.3 \\
\hline 6 & $61 / \mathrm{M}$ & $\mathrm{IOH}$ & 173 & 4 & 6,000 & 50 & 0.4 \\
\hline 7 & $70 / \mathbf{M}$ & $\mathrm{IOH}$ & 218 & 0 & $>6,000$ & 100 & 0.5 \\
\hline 8 & $60 / \mathrm{F}$ & $\mathrm{IOH}$ & 150 & 0 & 4,500 & 10 & 0.3 \\
\hline 9 & $67 / F$ & MSA & 210 & 5 & $>6,000$ & 70 & 0.3 \\
\hline 10 & $53 / \mathrm{F}$ & MSA & 143 & 9 & 1,800 & 20 & 0.2 \\
\hline 11 & $58 / \mathrm{F}$ & MSA & 146 & 8 & 2,000 & 25 & 0.3 \\
\hline 12 & $33 / F$ & ND & 20 & 0 & N.R.I & 35 & 0.3 \\
\hline Mean \pm SE & & & $105 \pm 22 \ddagger \ddagger$ & $2 \pm 1 \neq \ddagger$ & $5,400 \pm 850 \ddagger \ddagger$ & $39 \pm 7 \ddagger \ddagger$ & $0.34 \pm 0.02 \ddagger \ddagger$ \\
\hline Normal ${ }^{* *}$ & & & $368 \pm 24$ & $24 \pm 7$ & $2,800 \pm 250$ & $204 \pm 28$ & $5.2 \pm 1.8$ \\
\hline
\end{tabular}

* ND, noradrenergic defect. $¥ \mathrm{NE}$, plasma norepinephrine (picograms per milliliter). § Change in mean arterial pressure (torr) evoked by placement of hand in ice water for 1 min. "Dose (micrograms) of tyramine (Tyr) and phenylephrine (Phe) which raises mean arterial pressure 25 torr. Dose of isoproterenol (Iso) which lowers mean arterial pressure 25 torr. T No change in blood pressure with 6,000 $\mu \mathrm{g}$. ** Age-matched ( $n=8$ ). 拉 $P<0.01$ vs. normals.

defect was distinguished by levels of plasma norepinephrine at the lower limit of sensitivity of our assay $(20 \mathrm{pg} / \mathrm{ml})$, absence of pressor responsiveness to usual doses of tyramine, and a normal rise in heart rate in response to atropine. Elevated dopamine levels were associated with her low norepinephrine levels, suggesting a selective defect in norepinephrine synthesis or release (11).

A group of eight normal subjects were recruited to serve as controls. They ranged in age from 58 to $75 \mathrm{yr}$ and were admitted overnight before the study, remaining supine and fasted for the $8 \mathrm{~h}$ preceding the study.

All patients and normal subjects underwent baseline tests, including placement of a radial arterial line for blood pressure monitoring. This line was placed in the nondominant arm using the Seldinger technique and lidocaine infiltration at the wrist site.

Clonidine was administered while subjects were supine after an overnight fast. Blood pressure and heart rate were monitored with a Dinamap automated sphygmomanometer (Johnson \& Johnson Hospital Services, New Brunswick, NJ). After establishment of a stable baseline for $30 \mathrm{~min}$, clonidine was administered orally at a dose of $0.1 \mathrm{mg}$. If, after $2 \mathrm{~h}$, blood pressure had fallen, no additional clonidine was administered. If blood pressure was unchanged or increased at this time, an additional $0.2 \mathrm{mg}$ was given. $2 \mathrm{~h}$ later, according to the same criteria, an additional $0.4 \mathrm{mg}$ could be given and $2 \mathrm{~h}$ later, an additional $0.8 \mathrm{mg}$. Only two patients received $0.8 \mathrm{mg}$ as a single dose. Blood pressure was monitored for $4 \mathrm{~h}$ after the highest dose of clonidine in each patient.

Yohimbine was administered intravenously at doses from 4 to 64 $\mu \mathrm{g} / \mathrm{kg}$ as a 30 -s bolus. The studies were conducted under our Investigational New Drug exemption (18, 313; Food and Drug Administration) for use of yohimbine intravenously and orally. Yohimbine $\mathrm{HCl}$ was purchased from the Food and Drug Division of Sigma Chemical Co., St. Louis, Mo., and prepared for intravenous injection by dissolution in bacteriostatic saline $(0.5 \mathrm{mg} / \mathrm{ml})$ and filtration through a Millipore filter (Whatman, Inc., Clifton, NJ) $(0.22 \mu \mathrm{m})$. The first two subjects given yohimbine were studied supine. In four subjects (one with MSA and three with $\mathrm{IOH}$ ) a tilt table was used. In these subjects, a level of tilt was found that produced about a 30-torr fall in mean blood pressure, and could be tolerated by the patients for $10 \mathrm{~min}$ without evoking either syncope or a premonitory sign of syncope (maximum tolerated tilt [MTT]). This allowed observation of the immediate hemodynamic response to the postural stress and observation of any accommodation to this stress. Blood pressure, electrocardiogram, and heart rate were continuously monitored during yohimbine administration. Blood pressure was measured either directly with a radial arterial line or indirectly using a Dinamap automated sphygmomanometer. Samples for radioenzymatic measurement of plasma catecholamines were obtained at baseline, 20 min after saline (placebo) administration, and $20 \mathrm{~min}$ after administration of each of the cumulatively increasing doses of yohimbine.

Repeated measures analysis of variance was used to evaluate baseline and postdrug measurements. The comparisons of patients and controls (Table I) were made using unpaired $t$ tests.

\section{Results}

Clonidine administration. The responses of each of our subjects to clonidine are shown in Fig. 1. Six of the 12 subjects had pressor responses to clonidine, seen at doses of $0.1-0.8 \mathrm{mg}$. Of these patients, four had $\mathrm{IOH}$, one had MSA, and the last was the patient with the noradrenergic deficit. The remaining patients had average depressor responses of $18 \pm 3$ torr in response to the

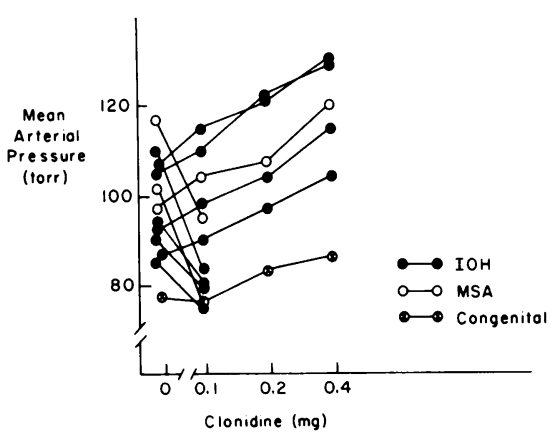

Figure 1. Influence of clonidine (0.1-0.4 mg, orally) on supine blood pressure in patients with autonomic dysfunction. Clonidine was administered after an overnight fast. Supine blood pressure and heart rate were monitored with a Dinamap automated sphygmomanometer. Shown are the peak changes in blood pressure 70-100 min after each dose. Each blood pressure determination is the mean of three consecutive sphygmomanometric recordings. Congenital, patient with congenital defect in noradrenergic neurotransmission. 
initial 0.1-mg dose and were not given any further doses. As shown in Fig. 2, there was a significant correlation between the response to $0.1 \mathrm{mg}$ clonidine and the supine plasma norepinephrine level. Those with the lowest supine norepinephrine levels tended to have a pressor response to clonidine and those with higher levels had a depressor response. No change in heart rate was noted in any patient after administration of $0.1 \mathrm{mg}$ clonidine. In three patients showing pressor responses, a 10-15beat per minute fall in heart rate was noted after the $0.4-\mathrm{mg}$ dose, indicating that vagal efferents were still functional.

Five individuals exhibiting a pressor response to oral clonidine were given a total of $0.7 \mathrm{mg}$ clonidine over a 4-h period. After the highest dose, all subjects experienced mild to severe hallucinations, feelings of depersonalization, or anxiety: Heart rate was not altered in any of these subjects, even during the hallucinations and dysphoria.

Yohimbine administration. Yohimbine was given to seven patients with autonomic dysfunction, three with $\mathrm{IOH}$, three with MSA, and the patient with the noradrenergic defect. Of these, three experienced pressor responses to clonidine, including the patient with the noradrenergic defect and one each with $\mathrm{IOH}$ and MSA. The influence of yohimbine on supine mean arterial pressure in these subjects is shown in Fig. 3. Patients with $\mathrm{IOH}$ and MSA were more sensitive to the pressor effects of yohimbine than were normal subjects. All had a pressor response to bolus doses as low as $4 \mu \mathrm{g} / \mathrm{kg}$. The pressor response was dose related and was associated with a measurable increment in plasma norepinephrine in those subjects with basal norepinephrine levels above $50 \mathrm{pg} / \mathrm{ml}$ (Fig. 4). Other patients had modest increments in plasma norepinephrine $(40 \pm 13-65 \pm 15 \mathrm{pg} / \mathrm{ml})$. The patient with the noradrenergic defect had a small decrease in pressure with yohimbine, perhaps related to an increment in dopamine release (11).

Fig. 5 shows the respective increments in plasma norepinephrine, epinephrine, and blood pressure in response to yo-

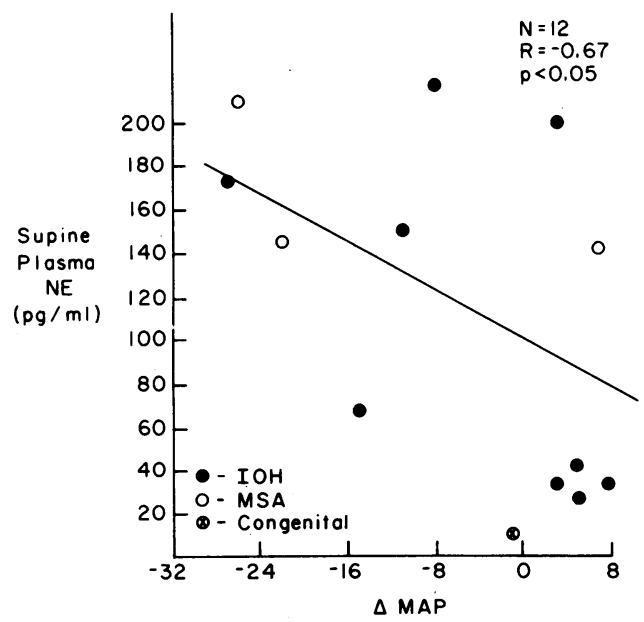

Figure 2. Relationship between the response to clonidine $(0.1 \mathrm{mg}$, orally) and supine plasma norepinephrine in patients with autonomic dysfunction. Each blood pressure determination is the mean of three consecutive sphygmomanometric recordings. Represented on the abscissa is the peak change in mean arterial pressure 70-100 min after clonidine. On the ordinate is the supine norepinephrine level measured radioenzymatically on another occasion. The solid line represents the least squares linear regression line calculated from the points.

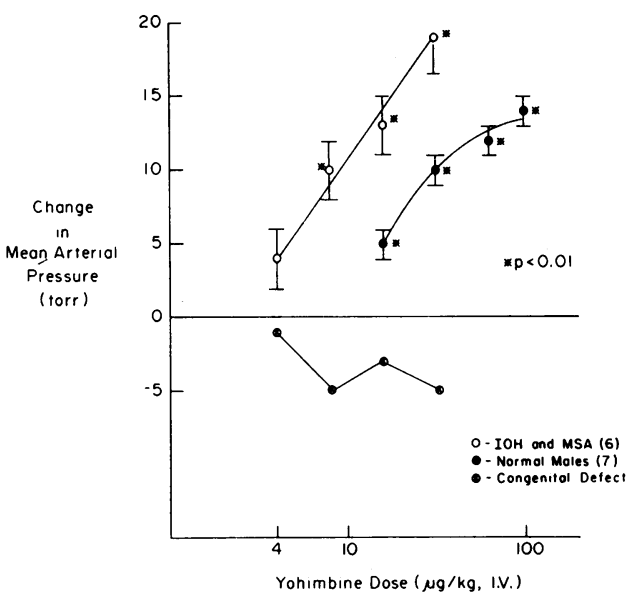

Figure 3. Influence of yohimbine (4-32 $\mu \mathrm{g} / \mathrm{kg}$, i.v.) on supine blood pressure in normal males, patients with degenerative autonomic dysfunction, and the patient with the congenital noradrenergic defect. Shown is the change in mean arterial pressure from baseline $20 \mathrm{~min}$ after each dose of yohimbine. Yohimbine was given in cumulative fashion so that the $32-\mu \mathrm{g} / \mathrm{kg}$ dose reflects a total dose of $60 \mu \mathrm{g} / \mathrm{kg}$. Statistical comparisons are between blood pressure before drug and after each dose.

himbine and the indirectly acting sympathomimetic amine, tyramine, in a 53-yr-old female with MSA (patient 10). Note that tyramine and yohimbine produced similar increments in blood pressure. However, tyramine elicited an increment predominantly in plasma epinephrine, and yohimbine produced an increment in plasma norepinephrine. This subject had demonstrated a pressor response to clonidine.

Fig. 6 shows intraarterial blood pressure tracings of the response to MTT of a 62-yr-old man with IOH (patient 5) before and after yohimbine at doses of 8 and $16 \mu \mathrm{g} / \mathrm{kg}$. Tilt from horizontal to 45 degrees lowered blood pressure from 110/57 to 85/ 40 over a 2 -min period. Pressure decreased further over the 10 min period of tilt to $45 / 20$ torr. In this subject, heart rate increased in response to MTT, although minimally in relation to the fall in blood pressure. Low doses of yohimbine modestly increased supine blood pressure but did not affect the initial fall in blood pressure during tilt. There was, however, a gradual recovery in blood pressure during the period of tilt to a final pressure of $112 / 50$. Increased yohimbine doses produced increments in supine blood pressure and further enhanced the ability to respond with a rise in pressure during MTT. At the end of

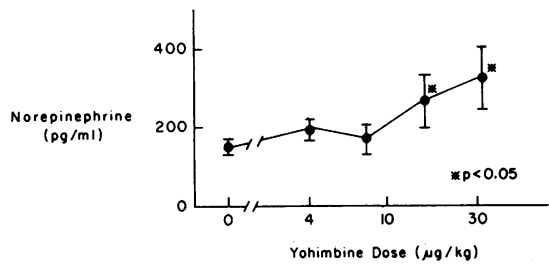

Figure 4. Influence of yohimbine on plasma norepinephrine in five subjects with autonomic dysfunction. The patient with the congenital defect who did not have a pressor response to yohimbine and a patient with $\mathrm{IOH}$ (subject 4) who had a baseline norepinephrine at the limit of detection of the radioenzymatic assay are not included. Statistical comparisons were made between baseline $(0 \mu \mathrm{g} / \mathrm{kg})$ and final levels of norepinephrine at each dose. 


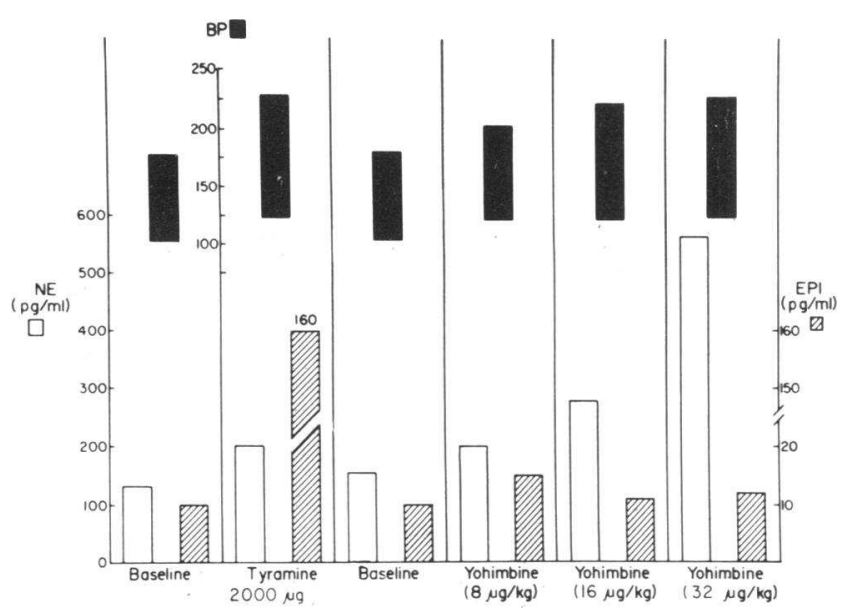

Figure 5. Comparison of the response to yohimbine $(8-32 \mu \mathrm{g} / \mathrm{kg})$ with the response to tyramine $(2,000 \mu \mathrm{g})$ in a patient (subject 10$)$ with multiple system atrophy. Note that yohimbine $(32 \mu \mathrm{g} / \mathrm{kg})$ and tyramine produced similar increments in systolic and diastolic pressure (black bars, upper scale). The pressor response to tyramine was associated with a 16-fold increment in epinephrine (EPI; hatched bars, righthand scale) and a 1.6-fold increment in norepinephrine (NE; open bars, left-hand scale) while yohimbine induced a 4.4-fold increment in norepinephrine and no change in epinephrine levels.

the study this subject was tilted to a point where he had previously complained of blurred vision and his blood pressure had dropped to $40 / 20$. He was able to tolerate this degree of tilt without symptoms after a cumulative dose of yohimbine of $108 \mu \mathrm{g} / \mathrm{kg}$. Fig. 7 shows that on the average, the four subjects exposed to tilt had a $50 \%$ reduction in the magnitude of their depressor response.
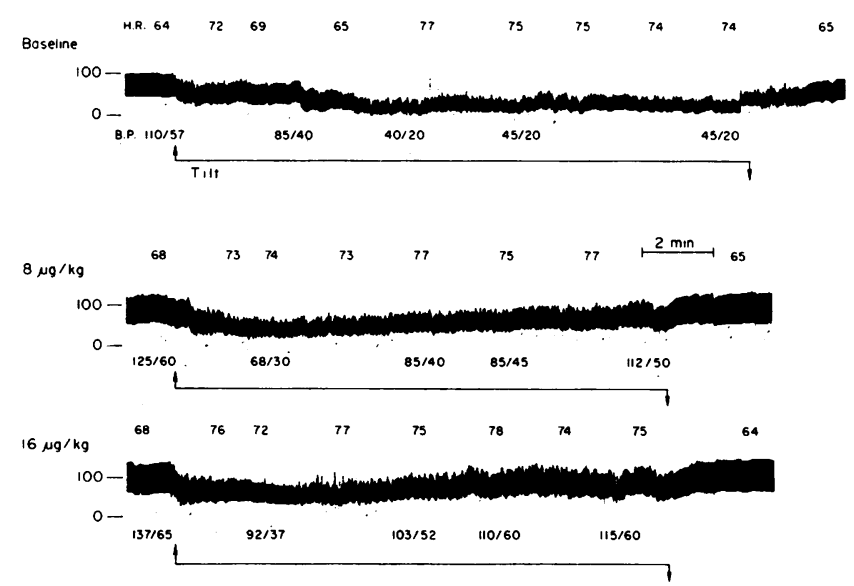

Figure 6. Intraarterial blood pressure recording showing serial responses to tilt of a patient with $\mathrm{IOH}$ (subject 5) before and after yohimbine ( 8 and $16 \mu \mathrm{g} / \mathrm{kg}$, i.v.). The time scale is shown on the middle panel. In each panel is shown the heart rate (H.R.) from the tachometer tracing, the blood pressure recording, and the blood pressure level (B.P.) read from this tracing. Yohimbine was administered as a 30-s bolus $5 \mathrm{~min}$ before these recordings began. The upward arrow under each tracing shows the time of onset of tilt. Tilting was performed over 20-30 s. The straight line under each tracing indicates the period during which tilt was maintained and the downward arrow indicates the time that the patient was returned to supine posture. The degree of tilt was the same for all these tracings.

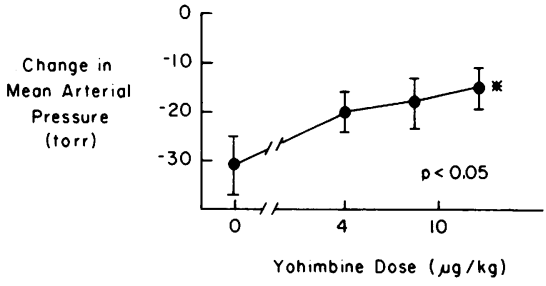

Figure 7. Influence of yohimbine (4-16 $\mu \mathrm{g} / \mathrm{kg}$, i.v.) on the response to tilt in four subjects studied on the tilt table. The abscissa represents the yohimbine dose. The ordinate represents the difference between supine mean arterial pressure and mean arterial pressure after $10 \mathrm{~min}$ of maximum tolerated tilt. Statistical comparisons were made between the fall in blood pressure in response to tilt before and after each dose of yohimbine.

\section{Discussion}

These studies show that, in general, an $\alpha_{2}$ partial agonist, clonidine, raises blood pressure in patients with severe autonomic insufficiency and lowers it in patients with less severe disease, when severity is defined in terms of the degree of abnormality in plasma norepinephrine level. Furthermore, the majority of severely affected patients can increase their sympathetic outflow in response to the $\alpha_{2}$-selective antagonist, yohimbine. Indeed, only one subject has failed to do so. The data suggest that, as shown in our previous report, clonidine may be an effective pressor agent in an occasional, severely affected patient (9). More importantly, yohimbine is potentially beneficial in treatment of patients with these disorders (14).

Several conclusions about the pathophysiology of acquired autonomic dysfunction are supported by these findings. The first of these is that some residual efferent sympathetic pathways are present even in the severely affected patients with IOH and MSA in our study. The pressor response to yohimbine in such patients and the depressor effects of clonidine in 6 of 11 patients further supports the concept that efferent pathways are at least partially intact in these individuals. Since yohimbine did not abolish the postural fall in blood pressure in these subjects, the data do not suggest that an excess of central $\alpha_{2}$ tone is of primary pathophysiological importance in the abnormal postural response of patients with $\mathrm{IOH}$ or MSA.

The actions of yohimbine and clonidine on sympathetic outflow could be due to effects on either central or peripheral sites. In previous studies, it has been difficult to demonstrate a peripheral effect of $\alpha_{2}$-adrenoreceptor agonists on catecholamine release rate in normal man, although techniques now available may facilitate this (15). Furthermore, experimental studies have shown that the effects of $\alpha_{2}$ agonists, like clonidine, on blood pressure can be entirely accounted for by a site of action in the central nervous system (8). It has also been difficult to show that the effects of yohimbine on blood pressure are due to peripheral effects. In fact, the association of the autonomic effects of yohimbine with changes in mood (7) and indices of central norepinephrine turnover $(10,16)$ are more suggestive of a central site of action. In the present study, we have no direct evidence to support either a central or peripheral site of action of clonidine in lowering blood pressure or of yohimbine in raising blood pressure in patients with autonomic dysfunction. 
Although blood pressure responses to yohimbine were noted at doses of 4 and $8 \mu \mathrm{g} / \mathrm{kg}$, the increment in plasma norepinephrine was not significant at these dose levels. This suggests that the small increase in norepinephrine release was sufficient to activate supersensitive $\alpha$-adrenoreceptors (Table I) but was not enough to be measured in peripheral blood.

Perhaps because of hypersensitivity development, it was not possible to predict the magnitude of the pressor response to yohimbine on the basis of the baseline supine plasma norepinephrine level. It might have been expected that the magnitude of the pressor response to yohimbine would have correlated with the respective effect of clonidine on blood pressure. This was not observed. There are several reasons for this. All patients (with the exception of the patient with the congenital defect in noradrenergic function) had a pressor response to yohimbine, whereas six exhibited a pressor response to clonidine and six had a depressor response. While there was an overall correlation of lower plasma norepinephrine with the pressor effects of both yohimbine and clonidine, patients with the very lowest plasma norepinephrine levels did not have as dramatic a degree of pressor effect to yohimbine as those with slightly higher (though still abnormally low) levels of norepinephrine. Yet four patients with the very lowest norepinephrine had the greatest pressor response to clonidine. It is this fact that prevents a correlation that might otherwise be present.

Another factor that may have contributed to the above disparity is the multiple potential levels at which yohimbine can act in patients with autonomic impairment. First, central $\alpha_{2}$ adrenoreceptors that tonically inhibit sympathetic outflow are blocked. Second, presynaptic $\alpha_{2}$-adrenoreceptors on peripheral noradrenergic neurons may be blocked. Third, postjunctional $\alpha_{2}$-adrenoreceptors mediating vasoconstriction may also be blocked by yohimbine. The latter two sites of action, but not necessarily the first, will be exposed to reduced circulating catecholamine levels in patients with autonomic impairment. In consequence, variable levels of hypersensitivity at the various sites may develop. The pressor effect of clonidine, on the other hand, will be mainly dependent on hypersensitivity of peripheral $\alpha_{2}$-adrenoreceptors alone.

Finally, the variable effect of adrenomedullary secretion may contribute to relatively different degrees of $\alpha_{1}$-hypersensitivity (upon which yohimbine's pressor effect ultimately depends) and $\alpha_{2}$-hypersensitivity (upon which clonidine's pressor effect mainly depends).

These findings provide an interesting therapeutic paradox for consideration. In at least two of our subjects, one with MSA and one with $\mathrm{IOH}$, we have data supporting the potential efficacy of both clonidine, an $\alpha_{2}$ agonist, and yohimbine, an $\alpha_{2}$ antagonist, as therapeutic pressor agents. This pharmacologic curiosity emphasizes the degree to which these pharmacologic probes can be used to define the extent of residual sympathetic outflow in these patients. These two individuals have so little sympathetic outflow that its inhibition by clonidine is obscured by supersensitivity to the peripheral pressor response to the agonist. At the same time, sympathetic outflow can be enhanced by the antagonist, and the norepinephrine released thereby is sufficient to raise blood pressure in these supersensitive subjects.

In conclusion, pharmacologic probes of the $\alpha_{2}$-adrenoreceptor are useful test agents in patients with severe autonomic dysfunction in discerning residual sympathetic tone. Clonidine, in particular, must be used with care, as sedation and psychosis are predictable side effects of aggressive dosing. Indeed, psychosis has also been reported in hypertensive patients treated with large doses of clonidine $(17,18)$. Since the doses that produced these effects are within the range of those used chronically to treat hypertension and since these effects are not generally thought to occur in the routine use of clonidine in hypertension, patients with autonomic dysfunction may be more sensitive to these undesirable actions of clonidine. In most cases the response to 0.1 $\mathrm{mg}$ is sufficient to demonstrate the degree to which residual sympathetic outflow helps maintain supine blood pressure. Yohimbine is useful in demonstrating that efferent pathways can be activated and, when used acutely, appears to have fewer side effects, suggesting it might have a place in the therapy of these subjects (14). The failure of yohimbine to increase blood pressure in the one patient with a deficiency in noradrenergic neurotransmission strongly supports our earlier conclusion that the pressor response to yohimbine is mediated by a drug-induced enhancement of sympathetic outflow (7).

\section{Acknowledgments}

The nurses of the Elliot V. Newman Clinical Research Center and the Vanderbilt Autonomic Dysfunction Clinic are thanked for the excellent care given to our patients. We particularly appreciate the help of Dawn Wade Kincaid, R.N., and Cheryl Parrish, R.N. We thank Mr. David Guy of the Physical Therapy Unit for use of the tilt table and Mr. Bolton Smith for bibliographic work. We also acknowledge Ms. Camille Morgan Huffines and Ms. Dorothea Boemer for preparation of the manuscript.

This work was supported by National Institutes of Health grants RR00095, GM15431, HL14192, and HL31419 and by a grant from the Dysautonomia Foundation. Dr. Goldberg and Dr. Hollister were Clinical Associate Physicians with the Elliot V. Newman Clinical Research Center. Dr. David Robertson is a Burroughs Wellcome Scholar in Clinical Pharmacology. Dr. Rose Marie Robertson is an Established Investigator of the American Heart Association.

\section{References}

1. Chobanian, A. V. 1982. Orthostatic hypotension. In Hypertension and Hypotension. H. R. Brunner and H. Gravas, editors. Marcel Dekker, New York. 435-454.

2. Thomas, J. E., A. Schirger, R. D. Fealey, and S. G. Sheps. 1982. Orthostatic hypotension. Mayo Clin. Proc. 56:117-125.

3. Ziegler, M: G. 1981. Choosing therapy for postural hypotension. Drug Ther. Rev. 6:49-60.

4. Ziegler, M. G., C. R. Lake, and I. J. Kopin. 1977. The sympatheticnervous system defect in primary orthostatic hypotension. $N$. Engl. J. Med. 296:293-297.

5. Polinsky, R. J., I. J. Kopin, M. H. Ebert, and V. Weise. 1981. Pharmacologic distinction of different orthostatic hypotension syndromes. Neurology. 31:1-7.

6. Onrot, J., I. Biaggioni, A. S. Hollister, D. Kincaid, and D. Robertson. 1985. Hemodynamic and humoral effects of caffeine in human autonomic failure. $N$. Engl. J. Med. 313:549-554.

7. Goldberg, M. R., A. S. Hollister, and D. Robertson. 1983. Influence of yohimbine on blood pressure autonomic reflexes and plasma catecholamines in man. Hypertension (Dallas). 5:77.2-778.

8. Kobinger, W. 1978. Central $\alpha$-adrenergic systems as targets for hypotensive drugs. Rev. Physiol. Biochem. Exp. Pharmacol. 81:39-100.

9. Robertson, D., M. R. Goldberg, A. S. Hollister, D. Wade, and R. M. Robertson. 1983. Clonidine raises blood pressure in severe idiopathic orthostatic hypotension. Am. J. Med. 74:193-200.

10. Goldberg, M. R., and D. Robertson. 1983. Yohimbine: a phar- 
macologic probe for study of the $\alpha_{2}$ adrenoreceptor. Pharmacol. Rev. 35:143-180.

11. Robertson, D., M. R. Goldberg, J. Onrot, A. S. Hollister, R. Wiley, J. G. Thompson, and R. M. Robertson. 1986. Isolated failure of autonomic noradrenergic neurotransmission: evidence for impaired $\beta$ hydroxylation of dopamine. N. Engl. J. Med. 314:1494-1497.

12. Robertson, D., A. S. Hollister, E. L. Carey, C. S. Tung, and M. R. Goldberg. 1984. Increased vascular beta-2 adrenoreceptor responsiveness in autonomic dysfunction. J. Am. Coll. Cardiol. 3:850856.

13. Laufer, S. T. 1942. Orthostatic hypotension (report of a case). Can. Med. Assoc. J. 46:160-164.

14. Brodde, O. E., M. Anlauf, J. Arroyo, R. Wagner, F. Weber, and
K. D. Buck. 1983. Hypersensitivity of adrenergic receptors and blood pressure response to oral yohimbine in orthostatic hypotension. $N$. Engl. J. Med. 308:1033-1034.

15. Goldstein, D. S., R. Zimlichman, R. Stull, H. R. Keiser, and I. J. Kopin. 1986. Estimation of intrasynaptic norepinephrine concentrations in man. Hypertension (Dallas). In press.

16. Charney, D. S., G. R. Heninger, and D. E. Sternberg. 1982. Assessment of $\alpha_{2}$ adrenergic autoreceptor function in humans: effects of oral yohimbine. Life Sci. 30:2033-2044.

17. Enoch, M. D., and G. E. M. Hammad. 1970. Acute hallucinosis due to clonidine. Curr. Med. Res. Opin. 4:670-671.

18. Elizur, A., and Z. Liberson. 1980. An acute psychotic episode at the beginning of clonidine therapy. Prog. Neuro-Psychopharmacol. 4: 211-213. 